\title{
Isotope shifts and zero-point motion of the nuclear surface
}

\author{
J. Dobaczewski* \\ W. K. Kellogg Radiation Laboratory, California Institute of Technology, Pasadena, California 91125 \\ P. Vogel and A. Winther ${ }^{\dagger}$ \\ Physics Department, California Institute of Technology, Pasadena, California 91125
}

(Received 15 November 1983)

\begin{abstract}
A simple procedure for correcting Hartree-Fock mean square radii for the effect of zero-point motion of the nuclear surface is proposed. The procedure has been applied to the calculation of the isotope shifts for even-even nuclei at the onset of deformation in the rare earth region, and a consistent improvement of agreement between the measured and calculated isotope shifts results. Preliminary results about the effect of pairing correlations on the nuclear radius have been also obtained. The effects seem to be significant, comparable with standard isotope shifts, and to depend on the effective two-body force used.
\end{abstract}

Systematic experimental data on the isotope shifts (IS) exist for elements throughout the periodic system. While on the average the nuclear radii are proportional to $A^{1 / 3}$, there are large variations due to nuclear structure effects. One of the more dramatic of these occurs at the onset of deformation in the rare earth region. The quantitative description of the isotope shifts in that region is the main topic of the present paper.

A comprehensive discussion of the theory of isotope shifts was given by Uher and Sorensen in $1966 .{ }^{1}$ Since then, the development of accurate Hartree-Fock calculations which allow for nonspherical equilibrium shapes has made a more detailed analysis possible. ${ }^{2}$ Such a description cannot be complete, however. Corrections to the Hartree-Fock density are expected to appear because the Hartree-Fock method includes only that part of the nuclear interaction which leads to the independent particle motion. The residual interactions lead most importantly to collective vibrations and corresponding ground state zero-point fluctuations of the nuclear density. There are two types of excitations which are relevant for the change in the mean square radius. These are the surface vibrations and the pairing vibrations, related respectively to the particle-hole and particleparticle components of the residual interaction. Both types of fluctuations can be determined from the microscopic random-phase approximation (RPA) calculations.

Corrections for these effects have already been discussed, for example by Campi and Epherre ${ }^{3}$ and by Reinhard and Drechsel. ${ }^{4}$ However, the prescriptions which have been used suffer from the fact that there are no clear rules to decide which surface vibrational modes should be included. In fact, if all surface degrees of freedom were included the simplest correction to the mean square radius would diverge. Recently a more systematic approach to the question of surface fluctuations has been given ${ }^{5}$ in terms of the HF and RPA methods. It is the aim of this paper to esti-

TABLE I. Experimental $B(E 2)^{1 / 2}$ and $B(E 3)^{1 / 2}$ values $^{\mathrm{a}}$ (in $e \mathrm{fm}^{\lambda}$ ), and the gap parameters $\Delta_{p}$ and $\Delta_{n}$ (in megaelectronvolts).

\begin{tabular}{|c|c|c|c|c|c|c|c|c|}
\hline \multirow[b]{2}{*}{$Z$} & \multirow[b]{2}{*}{$A$} & \multirow[b]{2}{*}{$0 \rightarrow 12$} & \multirow[b]{2}{*}{$0 \rightarrow 13$} & \multicolumn{2}{|c|}{$B(E \lambda, 0 \rightarrow n \lambda)^{1 / 2}$} & \multirow[b]{2}{*}{$0 \rightarrow 33$} & \multirow[b]{2}{*}{$\Delta_{p}$} & \multirow[b]{2}{*}{$\Delta_{n}$} \\
\hline & & & & $0 \rightarrow 22$ & $0 \rightarrow 23$ & & & \\
\hline \multirow[t]{5}{*}{$\mathrm{Nd}$} & 142 & $65(4)$ & $490(50)$ & & & & 1.23 & 1.37 \\
\hline & 144 & $69(2)$ & 510 & & & & 1.33 & 0.94 \\
\hline & 146 & $83(3)$ & $490(30)$ & & & & 1.32 & 1.02 \\
\hline & 148 & $116(2)$ & $330(90)$ & & & & 1.40 & 1.08 \\
\hline & 150 & $165(1)$ & $540(230)$ & & & & 1.06 & 1.10 \\
\hline \multirow[t]{5}{*}{$\mathrm{Sm}$} & 144 & $50(4)$ & 510 & & & & 1.26 & 1.42 \\
\hline & 148 & $84(1)$ & $590(40)$ & & & & 1.34 & 1.02 \\
\hline & 150 & $117(3)$ & $560(20)$ & & & & 1.43 & 1.13 \\
\hline & 152 & $186(2)$ & $350(40)$ & $29(3)$ & $260(40)$ & & 1.11 & 1.26 \\
\hline & 154 & $207(2)$ & $300(30)$ & $26(2)$ & & & 0.85 & 1.06 \\
\hline \multirow[t]{5}{*}{ Gd } & 152 & $140(5)$ & 560 & & 270 & & 1.48 & 1.06 \\
\hline & 154 & $196(1)$ & $460(80)$ & $34(2)$ & 220 & & 1.13 & 1.10 \\
\hline & 156 & $214(2)$ & $410(10)^{\mathrm{b}}$ & $33(1)^{b}$ & 210 & & 0.96 & 1.07 \\
\hline & 158 & $223(2)$ & $350(10)^{b}$ & $29(1)^{b}$ & 230 & $150^{\mathrm{b}}$ & 0.88 & 0.89 \\
\hline & 160 & $229(2)$ & $340(10)^{b}$ & $30(1)^{b}$ & 140 & 170 & 0.80 & 0.83 \\
\hline
\end{tabular}

aWhenever the experimental error is not given for a $\lambda=3$ transition a standard uncertainty of $10 \%$ is assumed. Data and uncertainties from corresponding most recent Nuclear Data Sheets.

bData from Ref. 7. 
mate the influence of the fluctuations on the nuclear radii based on HF results and the experimental information.

Surface vibrations give rise to a mean square fluctuation in the nuclear radius which is given by

$$
\sigma^{2}=\frac{R_{0}^{2}}{4 \pi} \sum_{n, \lambda} \beta_{\lambda}^{2}(n)
$$

where

$$
\beta_{\lambda}(n)=B(E \lambda, 0 \rightarrow n \lambda)^{1 / 2} /\left(\frac{3}{4 \pi} Z R_{0}^{\lambda}\right),
$$

$R_{0}$ is the nuclear radius, and $Z$ is the proton number. The $B(E \lambda, 0 \rightarrow n \lambda)$ values correspond to the excitation from the ground state to the mode $n$ with multipolarity $\lambda$.

One should not, however, use directly the mean square radius fluctuation $\sigma^{2}$, Eq. (1), as a correction of the Hartree-Fock radius because a major part of the zero-point fluctuations, the one which is related to excitations of the uncorrelated particle-hole states, $B(E \lambda, 0 \rightarrow p h)$, is already contained in the average field. The corrected mean square radius should thus have the form ${ }^{3,5}$

$$
\left\langle r^{2}\right\rangle=\left\langle r^{2}\right\rangle_{\mathrm{HF}}\left[1+\frac{5}{R_{0}^{2}}\left(\sigma^{2}-\sigma_{\mathrm{HF}}^{2}\right)\right],
$$

where $\sigma_{\mathrm{HF}}^{2}$ and $\sigma^{2}$ can be estimated from the Hartree-Fock,

$$
\left(B(E \lambda, 0 \rightarrow \lambda)_{\mathrm{HF}}\right)^{1 / 2}=\int \rho_{\mathrm{HF}} r^{\lambda} Y_{\lambda 0} d V,
$$

and experimental reduced transition probabilities, respectively. In this form our prescription is similar to the one used in Ref. 3 for the case of isotope shifts in the rubidium isotopes.

In practice, the difference $\sigma^{2}-\sigma_{\mathrm{HF}}^{2}$ receives contributions mainly from the low-lying states of multipolarity $\lambda=2,3$ (and perhaps 4). The contributions of the high-lying collective states (giant resonances), as well as the higher multipole states, to $\sigma^{2}$ are to a large extent canceled by the free particle-hole response (cf. Ref. 5). The contribution from the giant resonances is in fact quite negligible for the isotope shifts because of the smooth variation with mass number of the giant resonances and of the single particle response in the high energy region.

If the Hartree-Fock ground state is deformed one should leave out the $B\left(E 2,0 \rightarrow 2^{+}\right)$[and $B\left(E 4,0 \rightarrow 4^{+}\right)$] values for the rotational transitions. Since a nucleus which in a Hartree-Fock treatment has a small equilibrium deformation often exhibits large zero-point fluctuations around this nearly spherical shape it is, however, a better prescription to include also rotational transitions in $\sigma^{2}$ and subtract instead the corresponding $\mathrm{HF}$ expression.

For strongly deformed nuclei the leading order relation between the surface fluctuations and the $B(E \lambda)$ values, Eqs. (1) and (2), is no longer adequate and one needs a higher order expression relating the increase in the radius caused by a large deformation with the reduced multipole moments $M_{\lambda}$ [and thus $B(E \lambda)$ values] of the deformed charge distribution. Assuming the sharp-edge and uniform distribution of charge inside the surface defined by

$$
R(\theta, \phi)=\frac{R_{0}}{f(\beta)}\left(1+\sum_{\lambda} \beta_{\lambda} Y_{\lambda 0}(\theta, \phi)\right),
$$

where $f(\beta)$ enforces the volume conservation, one obtains up to the third order in $\beta_{\lambda}$

$$
\begin{aligned}
& \sigma^{2}=\frac{R_{0}^{2}}{4 \pi} \sum_{\lambda} \beta_{\lambda}\left(\beta_{\lambda}+\frac{5}{3} \sum_{\lambda^{\prime} \lambda^{\prime \prime}} \alpha_{\lambda \lambda^{\prime} \lambda^{\prime \prime}} \beta_{\lambda^{\prime}} \beta_{\lambda^{\prime \prime}}\right), \\
& M_{\lambda}=\beta_{\lambda}+\frac{1}{2}(\lambda+2) \sum_{\lambda^{\prime} \lambda^{\prime \prime}} \beta_{\lambda^{\prime}} \beta_{\lambda^{\prime \prime}} \alpha_{\lambda \lambda^{\prime} \lambda^{\prime \prime}} \\
&=B(E \lambda, 0 \rightarrow 1 \lambda)^{1 / 2}\left(\frac{3}{4 \pi} Z R_{0}^{\lambda}\right), \\
& \alpha_{\lambda \lambda^{\prime} \lambda^{\prime \prime}}=\left(\frac{(2 \lambda+1)\left(2 \lambda^{\prime}+1\right)\left(2 \lambda^{\prime \prime}+1\right)}{4 \pi}\right)^{1 / 2}\left(\begin{array}{ccc}
\lambda & \lambda^{\prime} & \lambda^{\prime \prime} \\
0 & 0 & 0
\end{array}\right)^{2}
\end{aligned}
$$

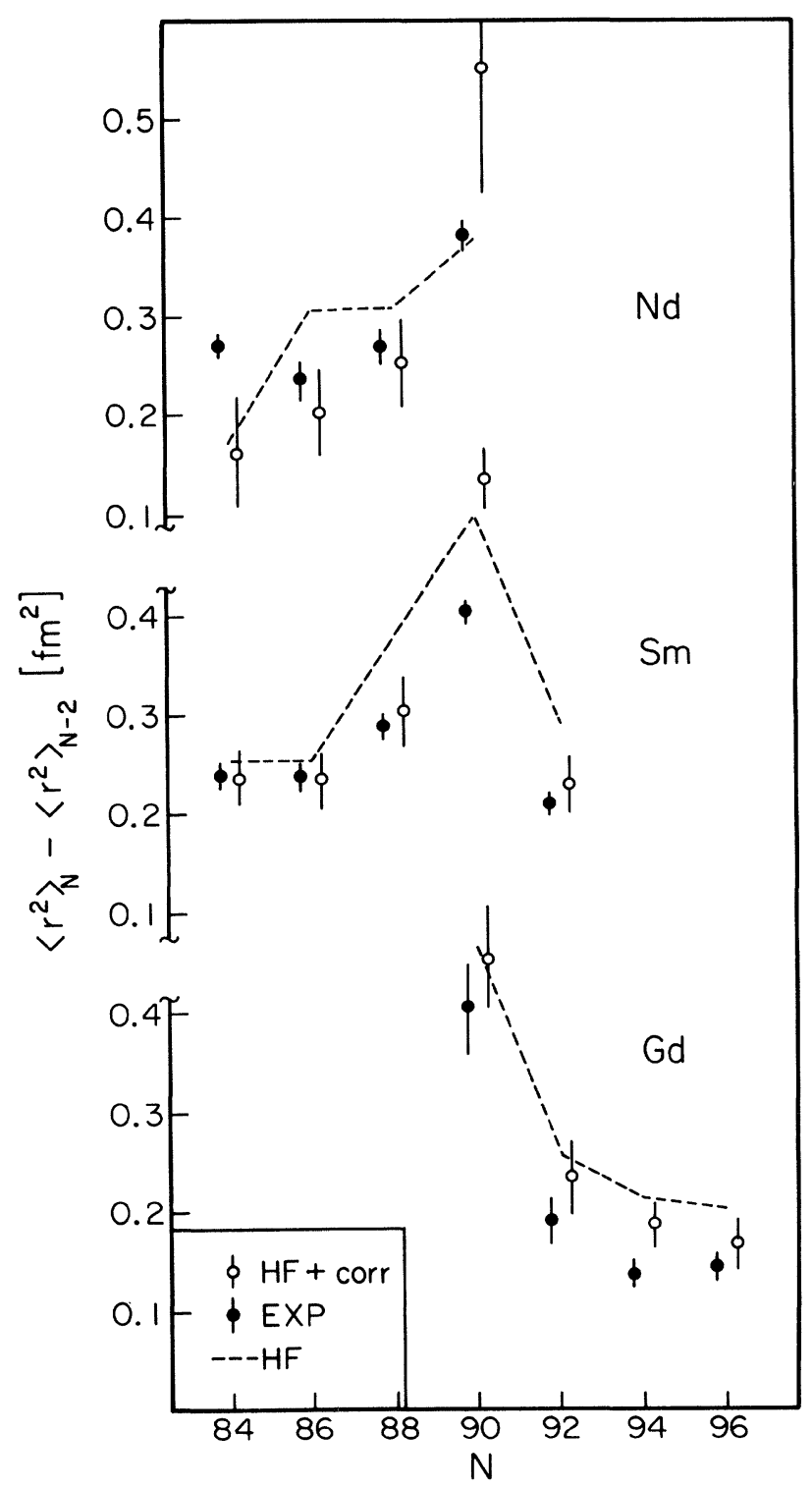

FIG. 1. Isotope shifts $\left\langle r^{2}\right\rangle_{N}-\left\langle r^{2}\right\rangle_{N-2}$ in $\mathrm{fm}^{2}$ for the even isotopes of the $\mathrm{Nd}, \mathrm{Sm}$, and $\mathrm{Gd}$ nuclei. Dashed line connects the values obtained in the deformed Hartree-Fock method. Full dots denote the experimental values (Ref. 8) while the open dots denote the Hartree-Fock values corrected for the zero-point surface fluctuations. The errors of the corrected isotope shifts result from the experimental errors of reduced transition probabilities (Table I). 
TABLE II. Mean square radii and isotope shifts (in $\mathrm{fm}^{2}$ ) of the $\mathrm{Nd}, \mathrm{Sm}$, and $\mathrm{Gd}$ isotopes.

\begin{tabular}{llllllll}
\hline \hline$Z$ & $A$ & $\left\langle r^{2}\right\rangle_{\mathrm{HF}}^{\mathrm{sph}}$ & $\left\langle r^{2}\right\rangle_{\mathrm{HF}}^{\mathrm{def}}$ & $\left\langle r^{2}\right\rangle_{\text {corr }}^{\mathrm{a}}$ & $\mathrm{IS}_{\mathrm{HF}}$ & $\mathrm{IS}_{\text {corr }}^{\mathrm{b}}$ & IS $_{\text {exp }}^{\mathrm{c}}$ \\
\hline \multirow{3}{*}{$\mathrm{Nd}$} & & & & & & \\
& 142 & 24.855 & 24.907 & 25.173 & & & \\
& 144 & 25.002 & 25.083 & 25.336 & 0.176 & $0.163(54)$ & $0.274(10)$ \\
& 146 & 25.164 & 25.392 & 25.539 & 0.309 & $0.203(43)$ & $0.239(16)$ \\
& 148 & 25.329 & 25.702 & 25.794 & 0.310 & $0.255(42)$ & $0.272(17)$ \\
& 150 & 25.492 & 26.081 & 26.346 & 0.379 & $0.522(125)$ & $0.385(15)$ \\
$\mathrm{Sm}$ & 144 & 25.182 & 25.229 & 25.444 & & & \\
& 148 & 25.487 & 25.739 & 25.917 & 0.510 & $0.473(35)$ & $0.479(25)$ \\
& 150 & 25.653 & 26.118 & 26.222 & 0.379 & $0.305(35)$ & $0.289(8)$ \\
& 152 & 25.806 & 26.618 & 26.760 & 0.500 & $0.538(32)$ & $0.403(10)$ \\
& 154 & 25.959 & 26.916 & 26.991 & 0.298 & $0.231(28)$ & $0.212(9)$ \\
& 152 & 25.980 & 26.492 & 26.684 & & & \\
& 154 & 26.128 & 26.959 & 27.139 & 0.467 & $0.455(53)$ & $0.407(45)$ \\
& 156 & 26.280 & 27.218 & 27.377 & 0.259 & $0.238(38)$ & $0.193(22)$ \\
& 158 & 26.439 & 27.432 & 27.566 & 0.214 & $0.189(25)$ & $0.137(10)$ \\
& 160 & 26.595 & 27.633 & 27.737 & 0.201 & $0.171(25)$ & $0.146(10)$ \\
& & & & & & \\
\hline \hline
\end{tabular}

aThe corrected mean square radii $\left\langle r^{2}\right\rangle_{\text {corr }}$ are calculated according to Eq. (3).

${ }^{\text {b}}$ The errors of $\mathrm{IS}_{\text {corr }}$ are related to the experimental errors of the $B(E \lambda)$ values (Table I) used in our correction procedure.

'Reference 8.

For a given set of experimental (or Hartree-Fock) transition rates $B(E \lambda, 0 \rightarrow 1 \lambda)$ we determine the deformation parameters $\beta_{\lambda}$, which play only an auxiliary role, and then the $\sigma^{2}$ (or $\sigma_{\mathrm{HF}}^{2}$ ) quantities. In principle, one could express $\sigma^{2}$ as an expansion in $M_{\lambda}$ up to the third order but this would give a much less accurate result than the expansion in $\beta_{\lambda}$. For not very collective states $(n>1)$ the leading order terms are adequate and are used in our numerical work below.

The procedure developed above was applied to the treatment of isotope shifts for nuclei with $82 \leqslant N \leqslant 96$ and $Z=60,62,64$ where one encounters large changes in quadrupole (and hexadecapole) deformations as well as relatively collective octupole states. The Hartree-Fock results were obtained with Skyrme III force and the reflection symmetry was imposed, i.e., $M_{3 \mathrm{HF}}=0$. The pairing correlations of the BCS type were included using the standard procedure. ${ }^{6}$ The equivalent radius $R_{0}$ has been calculated for each nucleus from the spherical Hartree-Fock mean square radius as $R_{0}=\left(5 / 3\left\langle r^{2}\right\rangle_{\mathrm{HF}, \mathrm{sph}}\right)^{1 / 2}$.

To calculate the correction part, $\sigma^{2}-\sigma_{\mathrm{HF}}^{2}$, we use experimental $B(E 2)$ values for the first (and sometimes second) $2^{+}$states and the $B(E 3)$ values for the low-lying $3^{-}$states. The $B(E \lambda)$ values used in the calculation are collected in Table I. For many nuclei $B(E 4)$ or $\beta_{4}$ values are not available. Because Hartree-Fock calculations generally successfully describe the experimental $\beta_{4}$ values, ${ }^{6}$ and because the contribution of $\lambda=4$ to Eq. (6) is small anyway, we have used $M_{4 \mathrm{HF}}$ for both $\sigma^{2}$ and $\sigma_{\mathrm{HF}}^{2}$ in Eq. (7).

The HF isotope shifts are compared with the experimental data in Fig. 1. While agreement appears to be quite good a closer inspection shows that the calculation systematically overestimates the isotope shifts.

The resulting corrections to the mean square charge radii are listed in Table II and the corresponding corrected isotope shifts are shown in Fig. 1. The improved agreement with respect to the HF results is obvious. The disagreement for the IS between ${ }^{152} \mathrm{Sm}$ and ${ }^{150} \mathrm{Sm}$ can probably be related to the inclusion of $n=2$ states in ${ }^{152} \mathrm{Sm}$ but not in ${ }^{150} \mathrm{Sm}$. The decrease in the zero-point quadrupole motion and the decrease in collectivity of the $3^{-}$states contribute about equally to the overall decrease of the isotope shift as com-

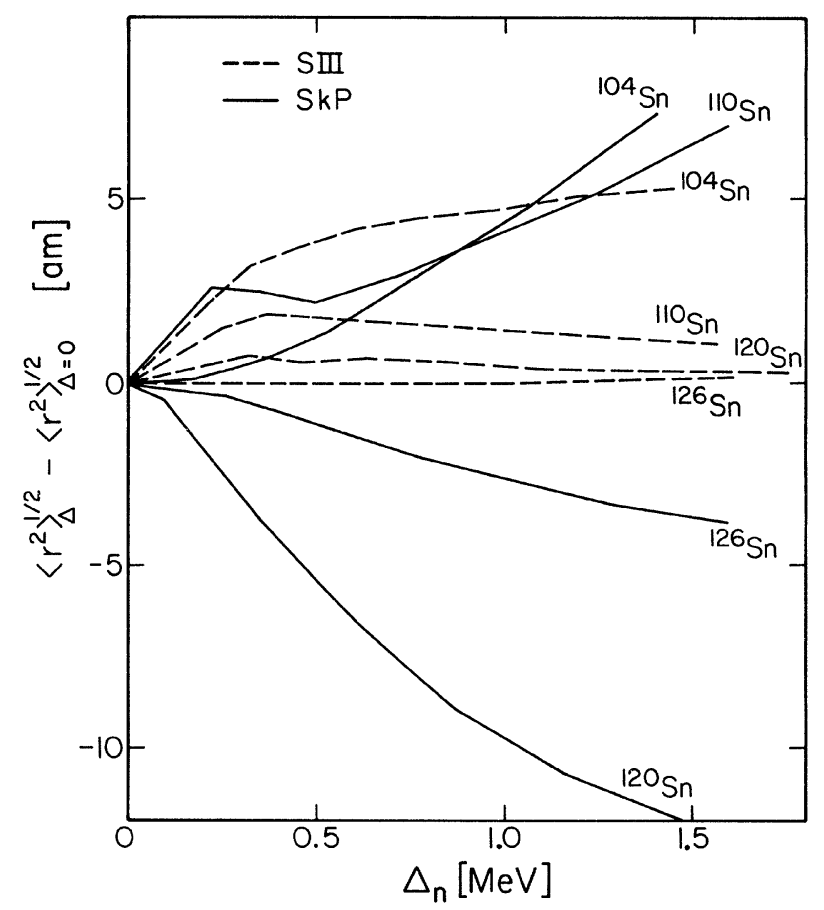

FIG. 2. Root mean square radii of the $\mathrm{Sn}$ isotopes as a function of the neutron pairing gap $\Delta_{n}$. The radii are obtained from the $\mathrm{HF}+\mathrm{BCS}$ calculations with the $S \mathrm{III}$ (dashed lines) and $\mathrm{SkP}^{9}$ (solid lines) Skyrme forces. The relative values with respect to the nonsuperfluid (HF) solutions are plotted. 
pared to the pure HF case.

The sharp surface approximation, which we used in deriving the formulas (6) and (7), can be tested by comparing the difference of the radii

$$
\Delta\left\langle r^{2}\right\rangle=\left\langle r^{2}\right\rangle_{\mathrm{HF}, \text { def }}-\left\langle r^{2}\right\rangle_{\mathrm{HF}, \mathrm{sph}}
$$

with the correction $5 \sigma_{\mathrm{HF}}^{2}$, which is determined from the Hartree-Fock multipole moments of the deformed nucleus. Such a comparison shows that both quantities agree within $10 \%$, even for $\beta_{2} \sim 0.3$ and $\Delta\left\langle r^{2}\right\rangle \sim 1 \mathrm{fm}^{2}$. It is essential, however, to use the higher-order corrections contained in Eqs. (6) and (7).

In the calculations which we have presented the effect of pairing was included through self-consistent BCS treatment. The corresponding gap parameters are given in Table I. In fact, pairing has an important effect on the nuclear radii and variations in the gap parameters will have, therefore, an important effect on the isotope shifts.

We have studied this effect numerically by artificially changing the neutron gap for series of spherical tin isotopes and the results are displayed in Fig. 2. Besides the Skyrme III force used previously the calculation was also performed with the Skyrme Pairing force. ${ }^{9}$ It is seen that depending on the two-body force used the pairing can have a significant effect on the nuclear radii and also on the isotope shifts.
The irregular behavior seen in Fig. 2 is associated with the $h_{11 / 2}$ neutron intruder state. Otherwise one would typically expect that the nuclear radius will increase with $\Delta$ as in the lightest $\mathrm{Sn}$ isotopes. The strong dependence on the two-body force shows that a definitive calculation of the isotope shifts can be done only when a force capable of adequate description of pairing has been developed. At the same time pairing should be included in a more consistent way than the BCS treatment of Ref. 6 . The reproduction of isotope shifts is one of the elements which should be taken into account in this endeavor. Once the self-consistent calculation of this kind is available it should be remembered that the corrections for the zero-point fluctuations of the pairing field should be included in a completely analogous way to the treatment above of the surface vibrations.

Finally, we notice that the pairing effect could be relevant for the until now unexplained odd-even staggering effect in isotope shifts. Some of the numerical calculations which we have performed indeed show an odd-even staggering of the correct magnitude.

This work was supported by the U.S. Department of Energy Grant No. DE-AT-03-81ER40002 and by the National Science Foundation under Grants No. PHY79-23638 and No. PHY82-07332.
"Permanent address: Institute of Theoretical Physics, Warsaw University, Hoza 69, PL-00681 Warsaw, Poland.

†Permanent address: Niels Bohr Institute, DK-2100 Copenhagen, Denmark.

${ }^{1}$ R. A. Uher and R. A. Sorensen, Nucl. Phys. 86, 1 (1966).

${ }^{2} \mathrm{X}$. Campi, in Nuclear Self-Consistent Fields, edited by G. Ripka and M. Porneuf (North-Holland, Amsterdam, 1975), p. 271.

${ }^{3}$ X. Campi and M. Epherre, Phys. Rev. C 22, 2605 (1980).
${ }^{4}$ P. G. Reinhard and D. Drechsel, Z. Phys. A 290,85 (1979).

${ }^{5}$ H. Esbensen and G. F. Bertsch, Phys. Rev. C 28, 355 (1983).

${ }^{6} \mathrm{H}$. Flocard, P. Quentin, and D. Vautherin, Phys. Lett. 46B, 30 (1973).

${ }^{7}$ F. K. McGowan and W. T. Milner, Phys. Rev. C 23, 1926 (1981).

${ }^{8}$ F. Boehm and P. L. Lee, At. Data Nucl. Data Tables 14, 605 (1974); K. Heilig and A. Steudel, ibid., 14, 613 (1974).

9J. Dobaczewski, H. Flocard, and J. Treiner (unpublished). 